\title{
Intercultivar Differences in Quality and Postharvest Life of Pomegranates Influenced by Partial Drying
}

\author{
Hassan A. Al-Kahtani \\ Food Science Department, P. O. Box 2460, King Saud University, Riyadh 11451 Saudi Arabia \\ Additional index words. Punica granatum, texture, sensory evaluation, decay, chemical analysis, storage life
}

Abstract. Eleven pomegranate (Punica granatum L.) cultivars were first evaluated, dried for 9 days at $20 \mathrm{C}$ and $47 \%$ relative humidity ( $\mathrm{RH}$ ), $30 \mathrm{C}$ and $33 \% \mathrm{RH}$, and $40 \mathrm{C}$ and $25 \% \mathrm{RH}$ before storage at $20 \pm 2 \mathrm{C}$ and $47 \%$ RH. 'TaifiA' was given the highest scores for sensory evaluation. 'Kab El-Jameel' contained significantly more edible portion and more juice, and had lower $\mathrm{pH}$ and higher acidity than any other cultivar. The highest vitamin $\mathrm{C}$ content was found in 'Taifi-A', 'Red Balady', and 'Mellasi'. Drying at 40C and $25 \%$ RH seriously damaged the pomegranates. 'De-Jativa, 'Molar', 'Succary', and 'Taifi-R' softened on the 4th day of drying and were more sensitive to drying conditions than the others. Drying at $30 \mathrm{C}$ and $33 \% \mathrm{RH}$ and at $20 \mathrm{C}$ and $47 \%$ RH did not appear to have visually deleterious effects on the internal portion of the fruit, but the edible portion was slightly inferior to that of fresh (refrigerated) fruits, particularly those dried at $30 \mathrm{C}$ and $33 \% \mathrm{RH}$. The juices of most dried fruits had higher pH, acidity, and total soluble solids content, but less vitamin $\mathrm{C}$ than fresh fruits. Fruits dried at $30 \mathrm{C}$ and $33 \% \mathrm{RH}$ or $20 \mathrm{C}$ and $47 \%$ RH remained acceptable at $20 \pm 2 \mathrm{C}$ and $47 \% \mathrm{RH}$ for up to 3 months or more, depending on the cultivar. Fungal decay (Aspergillus niger. Tiesh. and some Penicillum spp. were found) appeared only in fruits previously dried at $20 \mathrm{C}$ and $47 \%$ RH. Partial drying of pomegranates maybe useful for processed juice products.

Pomegranate fruits are mainly consumed fresh and as juice that can be used in beverages and for flavoring and coloring drinks, jam, jelly, and grenadine (Chace et al., 1930; Ewaidah, 1987; Hodgson, 1917; LaRue, 1969). 'Taifi' pomegranate is the most popular cultivar in Saudi Arabia, particularly in the South West region (Naser, 1983). However, some Egyptian and Spanish cultivars were introduced to the Riyadh region (Shaheen, 1985). The fresh fruits are only available from the middle of September until the beginning of November. Refrigeration has been recommended for storage and transport of pomegranates (Kader et al., 1984; Lutz and Hardenburg, 1977; Mukarjee, 1958; Pantastico et al., 1975). However, the high cost of refrigeration and disorders (mainly fungal growth and chilling injury symptoms) associated with fruit during refrigerated storage (Ben-Arie and Or, 1986; Elyatem and Kader, 1984) justify the search for an alternative method of preservation. Partial drying of pomegranate has not been commercially adopted, even though it was a normal practice of farmers and villagers in the past.

The objectives of this study were to evaluate postharvest quality of 11 pomegranate cultivars and to attempt to determine the effect of drying on their sensory, physical, and chemical properties.

\section{Materials and Methods}

Fruits. Fruits of all cultivars (Table 1) except 'Taifi-A' were obtained from 6- to 9-year-old trees grown at the Agricultural Experimental Station, College of Agriculture, King Saud Univ., Riyadh, during the 1989 harvest season. 'Taifi-A' fruits were harvested from a farm in Abha province in the southern region of Saudi Arabia and transported by air on the same day. The fruits were sorted for size and shape uniformity, and defective ones were eliminated. The fruits were then washed with tap water, kept in $0.5 \%$ sodium hypochlorite for 3 rein, rinsed with

Received for publication 1 Aug. 1990. Accepted for publication 21 May 1991. The cost of publishing this paper was defrayed in part by the payment of page charges. Under postal regulations, this paper therefore must be hereby marked advertisement solely to indicate this fact.
Table 1. Intercultivar differences' in fruit weight, percent edible portion, and percent juice.

\begin{tabular}{lrllll}
\hline \hline & \multicolumn{2}{c}{ Fruit $\mathrm{wt}(\mathrm{g})$} & & \multicolumn{2}{c}{ Percent by $\mathrm{wt}$} \\
\cline { 2 - 3 } \cline { 6 - 6 } Cultivar & Range & Mean & & Edible portion & Juice \\
\hline Taifi-A & $99-206$ & $160 \mathrm{f}$ & & $67.6 \mathrm{bc}$ & $52.3 \mathrm{~b}$ \\
Taifi-R & $232-264$ & $245 \mathrm{~b}-\mathrm{e}$ & & $59.1 \mathrm{de}$ & $43.0 \mathrm{~cd}$ \\
El-Madina & $191-277$ & $230 \mathrm{c}-\mathrm{f}$ & & $55.3 \mathrm{de}$ & $36.6 \mathrm{e}$ \\
Kab El-Jameel & $312-364$ & $305 \mathrm{ab}$ & & $77.2 \mathrm{a}$ & $57.0 \mathrm{a}$ \\
Red Balady & $220-299$ & $266 \mathrm{a}-\mathrm{d}$ & & $61.2 \mathrm{~cd}$ & $43.0 \mathrm{~cd}$ \\
Mellasi & $253-428$ & $325 \mathrm{a}$ & & $61.6 \mathrm{~cd}$ & $42.0 \mathrm{~d}$ \\
De-Jativa & $163-295$ & $219 \mathrm{cef}$ & & $57.8 \mathrm{de}$ & $43.5 \mathrm{~cd}$ \\
Molar & $156-198$ & $173 \mathrm{ef}$ & & $61.7 \mathrm{~cd}$ & $42.5 \mathrm{~d}$ \\
Manfaloti & $305-379$ & $323 \mathrm{a}$ & & $72.4 \mathrm{ab}$ & $47.4 \mathrm{c}$ \\
Banati & $232-332$ & $293 \mathrm{abc}$ & & $58.7 \mathrm{de}$ & $41.1 \mathrm{de}$ \\
Succary & $133-205$ & $167 \mathrm{f}$ & & $54.2 \mathrm{e}$ & $41.2 \mathrm{de}$ \\
\hline
\end{tabular}

${ }^{2}$ Means separation in columns by Duncan's multiple range test, $P=$ 0.05 .

tap water, dried with paper tissue, and held at 5C and 90\% $\mathrm{RH}$ until the next morning. Some fresh fruits from each cultivar were held at 5C for later use for comparison with the dried fruits, while some fruit were used for quality evaluation.

Quality evaluation of fresh fruits, sample preparation, and chemical analysis. Ten fresh fruits of each cultivar were weighed and hand cracked, and the arils were separated from other tissues. Half of the edible portion was used for sensory evaluation, while the juice of the other half was extracted using an electric extractor (type 140.6.03, model Depose, Moulinx, France). The juice from individual fruit was pooled, filtered through cheesecloth, and used for chemical analysis. The juice and edible portion were weighed separately and weights of each cultivar were obtained. The chemical analysis of the juice included determination of $\mathrm{pH}$ (model 6000, Sargent-Welch, Skokie, Ill.), soluble solids concentration (TSS; Abbe refractometer, Bausch and Lomb, model 10480, Cambridge Instrument, Buffalo, N.Y.), titratable acidity (percent citric acid), vitamin $\mathrm{C}(\mathrm{mg} / 100 \mathrm{ml})$, and reducing or nonreducing and total sugars (AOAC, 1980).

Sensory analysis. A 20-member untrained panel participated in sensory evaluation. The edible portion of fruits was given to 
Table 2. Sensory evaluation of pomegranate fruits of different cultivars. Means of 20 judges $\pm \mathrm{SE}^{2}$.

\begin{tabular}{llccc}
\hline \hline & \multicolumn{4}{c}{ Sensory scores } \\
\cline { 2 - 5 } Cultivar & \multicolumn{1}{c}{ Color } & Flavor & Mouth feel & $\begin{array}{c}\text { Overall } \\
\text { acceptability }\end{array}$ \\
\hline Taifi-A & $8.8 \pm 0.40 \mathrm{a}$ & $7.5 \pm 0.5 \mathrm{a}$ & $7.9 \pm 0.3 \mathrm{a}$ & $8.6 \pm 0.2 \mathrm{a}$ \\
Taifi-R & $2.4 \pm 0.3 \mathrm{~g}$ & $6.9 \pm 0.4 \mathrm{ab}$ & $6.9 \pm 0.4 \mathrm{ab}$ & $6.0 \pm 0.4 \mathrm{~b}$ \\
El-Madina & $2.8 \pm 0.3 \mathrm{fg}$ & $4.4 \pm 0.5 \mathrm{de}$ & $3.6 \pm 0.5 \mathrm{f}$ & $4.7 \pm 0.5 \mathrm{~cd}$ \\
Khab El-Jameel & $7.4 \pm 0.3 \mathrm{bc}$ & $3.6 \pm 0.3 \mathrm{ef}$ & $5.1 \pm 0.5 \mathrm{~cd}$ & $5.4 \pm 0.4 \mathrm{bc}$ \\
Red Balady & $7.8 \pm 0.2 \mathrm{~b}$ & $3.2 \pm 0.5 \mathrm{e}$ & $3.7 \pm 0.5 \mathrm{ef}$ & $4.2 \pm 0.5 \mathrm{~d}$ \\
Mellasi & $4.6 \pm 0.3 \mathrm{~d}$ & $4.7 \pm 0.3 \mathrm{de}$ & $4.9 \pm 0.3 \mathrm{de}$ & $5.0 \pm 0.3 \mathrm{bcd}$ \\
De-Jative & $3.7 \pm 0.4 \mathrm{e}$ & $6.2 \pm 0.4 \mathrm{bc}$ & $7.5 \pm 0.4 \mathrm{ab}$ & $6.0 \pm 0.5 \mathrm{~b}$ \\
Molar & $3.6 \pm 0.3 \mathrm{ef}$ & $6.3 \pm 0.4 \mathrm{~b}$ & $6.3 \pm 0.6 \mathrm{bc}$ & $6.0 \pm 0.4 \mathrm{~b}$ \\
Manfaloti & $6.5 \pm 0.4 \mathrm{c}$ & $5.0 \pm 0.4 \mathrm{~d}$ & $4.8 \pm 0.5 \mathrm{def}$ & $5.5 \pm 0.4 \mathrm{bc}$ \\
Banati & $4.0 \pm 0.3 \mathrm{de}$ & $4.6 \pm 0.3 \mathrm{de}$ & $4.9 \pm 0.4 \mathrm{def}$ & $4.7 \pm 0.4 \mathrm{~cd}$ \\
Succary & $3.4 \pm 0.2 \mathrm{ef}$ & $5.0 \pm 0.4 \mathrm{~cd}$ & $5.4 \pm 0.5 \mathrm{~cd}$ & $5.3 \pm 0.3 \mathrm{bcd}$ \\
\hline
\end{tabular}

${ }^{2}$ Means separation in columns by Duncan's multiple range test, $P=0.05$.

'Each factor was graded on a 10 -point scale $(1=$ poor, $10=$ best $)$.

Table 3. Effect of drying conditions on weight loss of pomegranates under various temperature/relative humidity conditions [vapor pressure difference $(\mathrm{kPa})]$.

\begin{tabular}{|c|c|c|c|}
\hline \multirow[b]{2}{*}{ Cultivar } & \multicolumn{3}{|c|}{ Wt $\operatorname{loss}^{2}(\%)$ after 9 days at } \\
\hline & $\begin{array}{c}20 \mathrm{C}, 47 \% \mathrm{RH} \\
(1.2)\end{array}$ & $\begin{array}{l}30 \mathrm{C}, 33 \% \mathrm{RH} \\
(2.9)\end{array}$ & $\begin{array}{c}40 \mathrm{C}, 25 \% \mathrm{RH} \\
(5.5)\end{array}$ \\
\hline Taifi-A & $9.3 \mathrm{e}$ & $12.3 \mathrm{f}$ & $.24 .0 \mathrm{e}$ \\
\hline Taifi-R & $14.7 \mathrm{~d}$ & $19.6 \mathrm{de}$ & $29.6 \mathrm{~cd}$ \\
\hline El-Madina & $17.3 \mathrm{bc}$ & $21.8 \mathrm{c}$ & $25.5 \mathrm{e}$ \\
\hline Kab El-Jameel & $14.8 \mathrm{~d}$ & $19.5 \mathrm{de}$ & $28.4 \mathrm{~d}$ \\
\hline Red Balady & $17.6 \mathrm{~b}$ & $18.8 \mathrm{e}$ & $30.3 \mathrm{c}$ \\
\hline Mellasi & $14.3 \mathrm{~d}$ & $19.9 \mathrm{de}$ & $24.7 \mathrm{e}$ \\
\hline De-Jetiva & $18.3 \mathrm{~b}$ & $22.7 \mathrm{C}$ & $37.6 \mathrm{~b}$ \\
\hline Molar & $21.3 \mathrm{a}$ & $24.3 \mathrm{~b}$ & $40.2 \mathrm{a}$ \\
\hline Manfaloti & $18.2 \mathrm{~b}$ & $20.2 \mathrm{~d}$ & $25.8 \mathrm{e}$ \\
\hline Banati & $15.9 \mathrm{~cd}$ & $22.1 \mathrm{c}$ & $25.2 \mathrm{e}$ \\
\hline Succary & $20.1 \mathrm{a}$ & $27.4 \mathrm{a}$ & $39.6 \mathrm{a}$ \\
\hline \multicolumn{4}{|c|}{$\begin{array}{l}{ }^{2} \text { Means separation in columns by Duncan's multiple range test, } P= \\
0.05 \text {. }\end{array}$} \\
\hline \multicolumn{4}{|c|}{$\begin{array}{l}\text { Table 4. Percentage of discarded pomegranates after drying for } 9 \\
\text { days at different drying conditions. }\end{array}$} \\
\hline \multicolumn{4}{|c|}{ Drying temp $\left({ }^{\circ} \mathrm{C}\right) /$ relative humidity $(\%)$} \\
\hline \multirow{3}{*}{ Cultivar } & $20 / 47$ & $30 / 33$ & $40 / 25$ \\
\hline & $(1.2)$ & $(2.9)$ & $(5.5)$ \\
\hline & \multicolumn{3}{|c|}{ Vapor pressure difference $(\mathrm{kPa})$} \\
\hline Taifi-A & $0.5 \mathrm{~cd}$ & $3.0 \mathrm{~d}$ & $68 \mathrm{~b}$ \\
\hline Taifi-R & $1.0 \mathrm{~cd}$ & $9.0 \mathrm{bc}$ & $89 \mathrm{a}$ \\
\hline El-Madina & $0.0 \mathrm{~d}$ & $3.0 \mathrm{~d}$ & $67 \mathrm{~b}$ \\
\hline Khab El-Jameel & $0.0 \mathrm{~d}$ & $0.5 \mathrm{~d}$ & $59 \mathrm{~d}$ \\
\hline Red Balady & $2.0 \mathrm{bc}$ & $4.5 \mathrm{~cd}$ & $63 \mathrm{~b}$ \\
\hline Mellasi & $0.0 \mathrm{~d}$ & $2.5 \mathrm{~d}$ & $55 \mathrm{~b}$ \\
\hline De-Jativa & $4.0 \mathrm{a}$ & $18.0 \mathrm{a}$ & $95 \mathrm{a}$ \\
\hline Molar & $3.5 \mathrm{ab}$ & $16.0 \mathrm{a}$ & $88 \mathrm{a}$ \\
\hline Manfaloti & $0.0 \mathrm{~d}$ & $2.0 \mathrm{~d}$ & $57 \mathrm{~b}$ \\
\hline Banati & $0.0 \mathrm{~d}$ & $1.5 \mathrm{~d}$ & $67 \mathrm{~b}$ \\
\hline Succary & $1.5 \mathrm{~cd}$ & $13.5 \mathrm{ab}$ & $92 \mathrm{a}$ \\
\hline
\end{tabular}

'Means separation in columns by Duncan's multiple range test, $P=$ 0.05 .

each panelist and a 10-point hedonic scale $(1=$ poor; $10=$ best) was used to evaluate color, flavor, mouth feel, and overall acceptability. The scores were then calculated for each cultivar.

Texture. After removal of the calyx, five fruits per cultivar were compressed on their equator by use of an Instron Universal
Table 5. Compressive strength and strain as measurements' of fresh (cold-stored) and dried pomegranate fruits texture.

\begin{tabular}{|c|c|c|c|c|}
\hline \multirow[b]{3}{*}{ Cultivar } & \multicolumn{4}{|c|}{ Fruit } \\
\hline & & \multicolumn{3}{|c|}{ Dried $\left[{ }^{\circ} \mathrm{C} / \% \mathrm{RH}(\mathrm{VPD})^{y}\right]$} \\
\hline & Fresh & $\begin{array}{c}20 / 47 \\
(1.2)\end{array}$ & $\begin{array}{c}30 / 33 \\
(2.9)\end{array}$ & $\begin{array}{c}40 / 25 \\
(5.5)\end{array}$ \\
\hline & \multicolumn{4}{|c|}{ Compressive strength $\left(\mathrm{kg} \cdot \mathrm{cm}^{-2}\right)$} \\
\hline Taifi-A & $2.0 \mathrm{bc}$ & $3.5 \mathrm{a}$ & $1.2 \mathrm{f}$ & $1.0 \mathrm{~g}$ \\
\hline Taifi-R & $1.9 \mathrm{bc}$ & $2.3 \mathrm{~cd}$ & $2.8 \mathrm{~cd}$ & $1.7 \mathrm{f}$ \\
\hline El-Madina & $1.8 \mathrm{bcd}$ & $1.5 \mathrm{c}$ & $2.2 \mathrm{e}$ & $2.5 \mathrm{bcd}$ \\
\hline Kab El-Jameel & $2.0 \mathrm{bc}$ & $2.0 \mathrm{~d}$ & $2.1 \mathrm{e}$ & $3.0 \mathrm{a}$ \\
\hline Red Balady & $1.5 \mathrm{de}$ & $2.5 \mathrm{c}$ & $2.9 \mathrm{c}$ & $2.1 \mathrm{def}$ \\
\hline Mellasi & $2.2 \mathrm{ab}$ & $2.0 \mathrm{~d}$ & $2.4 \mathrm{de}$ & $2.1 \mathrm{de}$ \\
\hline De-Jativa & $1.9 \mathrm{bc}$ & $1.6 \mathrm{c}$ & $2.2 \mathrm{e}$ & $2.1 \mathrm{def}$ \\
\hline Molar & $1.4 \mathrm{e}$ & $2.0 \mathrm{~d}$ & $1.6 \mathrm{f}$ & $2.9 \mathrm{ab}$ \\
\hline Manfaloti & $1.7 \mathrm{cde}$ & $1.8 \mathrm{de}$ & $4.9 \mathrm{a}$ & 2.4 cde \\
\hline Banati & $2.4 \mathrm{a}$ & $1.6 \mathrm{e}$ & $3.5 \mathrm{~b}$ & $2.6 \mathrm{abc}$ \\
\hline \multirow[t]{2}{*}{ Succary } & $1.7 \mathrm{cde}$ & $3.0 \mathrm{~b}$ & $2.1 \mathrm{c}$ & 2.1 ef \\
\hline & \multicolumn{4}{|c|}{ Compressive strain (\%) } \\
\hline Taifi-A & $331 \mathrm{c}$ & $336 \mathrm{bc}$ & $168 \mathrm{f}$ & $137 \mathrm{~g}$ \\
\hline Taifi-R & 319 ed & $356 \mathrm{bcd}$ & $124 \mathrm{~g}$ & $372 \mathrm{c}$ \\
\hline El-Madina & $337 \mathrm{c}$ & $347 \mathrm{bcd}$ & $302 \mathrm{~b}$ & 189 ef \\
\hline Kab El-Jameel & $268 \mathrm{e}$ & $232 \mathrm{f}$ & $166 \mathrm{f}$ & $142 \mathrm{~g}$ \\
\hline Red Balady & $277 \mathrm{de}$ & $308 \mathrm{de}$ & $266 \mathrm{c}$ & $242 \mathrm{~d}$ \\
\hline Mellasi & $321 \mathrm{~cd}$ & $276 \mathrm{ef}$ & $214 \mathrm{e}$ & $151 \mathrm{~g}$ \\
\hline De-Jativa & $449 \mathrm{a}$ & $440 \mathrm{a}$ & $313 b$ & $497 \mathrm{a}$ \\
\hline Molar & $331 \mathrm{c}$ & $398 \mathrm{ab}$ & $159 \mathrm{f}$ & 210 de \\
\hline Manfaloti & 300 cde & $237 \mathrm{f}$ & $231 \mathrm{de}$ & $170 \mathrm{fg}$ \\
\hline Banati & $319 \mathrm{~cd}$ & 259 ef & $255 \mathrm{~cd}$ & $209 \mathrm{de}$ \\
\hline Succary & $400 \mathrm{~b}$ & 346 ed & $424 \mathrm{a}$ & $459 \mathrm{~b}$ \\
\hline
\end{tabular}

${ }^{2}$ Means (of five fruits) separation in columns by Duncan's multiple range test, $P=0.05$.

${ }^{y} \mathrm{VPD}=$ vapor pressure difference $(\mathrm{kPa})$.

Testing Machine (Model 1197, Buckinghamshire, England) with compression plate diameter of $100 \mathrm{~mm}$. Crosshead speed was $5 \mathrm{~mm} \cdot \mathrm{min}^{-1}$ and the accuracy was $\pm 1 \mathrm{~g}$. Total compressive distance, initial height, contact area, and the force necessary to cause cracking of the fruit were recorded. Both compressive strength (kilograms per square centimeter) and compressive strain (percent) were calculated.

Drying of pomegranates. The fruits of each cultivar were divided into three groups. One group was left to dry for 9 days at room temperature $(\approx 20 \mathrm{C})$ and $47 \% \mathrm{RH}$. The second and third groups were dried for 9 days at $30 \mathrm{C}$ and $33 \% \mathrm{RH}$ or at $40 \mathrm{C}$ and $25 \% \mathrm{RH}$, using drying ovens. The dried fruits were then 
Table 6. Number of panel members whose score' differed for refrigerated and fruit dried for 9 days under several drying conditions $[\mathrm{T}=$ temperature $(\mathrm{C}), \mathrm{RH}=$ relative humidity $(\%), \mathrm{VPD}=$ vapor pressure difference $(\mathrm{kPa})]$.

\begin{tabular}{|c|c|c|c|c|c|c|c|c|c|}
\hline \multirow[b]{2}{*}{ Cultivar } & \multicolumn{3}{|c|}{ Color } & \multicolumn{3}{|c|}{ Flavor } & \multicolumn{3}{|c|}{ Overall acceptability } \\
\hline & $\begin{array}{l}20 \\
47 \\
1.2 \\
\end{array}$ & $\begin{array}{r}30 \\
33 \\
2.9 \\
\end{array}$ & $\begin{array}{r}40 \\
25 \\
5.5 \\
\end{array}$ & $\begin{array}{l}20 \\
47 \\
1.2 \\
\end{array}$ & $\begin{array}{r}30 \\
33 \\
2.9 \\
\end{array}$ & $\begin{array}{l}40 \\
25 \\
5.5 \\
\end{array}$ & $\begin{array}{c}20 \\
47 \\
1.2\end{array}$ & $\begin{array}{l}30 \\
33 \\
2.9 \\
\end{array}$ & $\begin{array}{l}40 \\
25 \\
5.5 \\
\end{array}$ \\
\hline Taifia-A & $0.5 \mathrm{ab}$ & $2.0 \mathrm{~cd}$ & $19.5 \mathrm{a}$ & $1.5 \mathrm{~b}$ & $1.5 \mathrm{~b}$ & $19.5 \mathrm{a}$ & $2.0 \mathrm{~b}$ & $1.0 \mathrm{~cd}$ & $20.0 \mathrm{a}$ \\
\hline Taifi-R & $0.0 \mathrm{~b}$ & $4.0 \mathrm{c}$ & $20.0 \mathrm{a}$ & $2.0 \mathrm{~b}$ & $2.5 \mathrm{~b}$ & $17.0 \mathrm{ab}$ & $2.5 \mathrm{~b}$ & $3.5 \mathrm{bc}$ & $19.0 \mathrm{a}$ \\
\hline El-Madina & $0.5 \mathrm{ab}$ & $2.5 \mathrm{~cd}$ & $19.0 \mathrm{a}$ & $1.5 \mathrm{~b}$ & $1.0 \mathrm{~b}$ & $14.5 \mathrm{bc}$ & $0.5 \mathrm{~b}$ & $0.0 \mathrm{~b}$ & $17.5 \mathrm{a}$ \\
\hline Khab El-Jameel & $1.0 \mathrm{ab}$ & $3.0 \mathrm{~cd}$ & $20.0 \mathrm{a}$ & $1.0 \mathrm{~b}$ & $2.0 \mathrm{~b}$ & $18.5 \mathrm{ab}$ & $1.0 \mathrm{~b}$ & $3.0 \mathrm{c}$ & $18.0 \mathrm{a}$ \\
\hline Red Balady & $0.0 \mathrm{~b}$ & $2.0 \mathrm{~cd}$ & $20.0 \mathrm{a}$ & $1.5 \mathrm{~b}$ & $0.5 \mathrm{~b}$ & $16.5 \mathrm{ab}$ & $2.0 \mathrm{~b}$ & $1.5 \mathrm{~cd}$ & $19.5 \mathrm{a}$ \\
\hline Mellasi & $0.5 \mathrm{ab}$ & $0.0 \mathrm{~d}$ & $20.0 \mathrm{a}$ & $2.0 \mathrm{~b}$ & $1.0 \mathrm{~b}$ & $15.0 \mathrm{abc}$ & $3.5 \mathrm{~b}$ & $2.0 \mathrm{~cd}$ & $18.0 \mathrm{a}$ \\
\hline De-Jativa & $2.0 \mathrm{a}$ & $7.5 \mathrm{ab}$ & $19.5 \mathrm{a}$ & $8.0 \mathrm{a}$ & $11.0 \mathrm{a}$ & $19.5 \mathrm{a}$ & $8.5 \mathrm{a}$ & $10.5 \mathrm{a}$ & $20.0 \mathrm{a}$ \\
\hline Molar & $1.5 \mathrm{ab}$ & $8.5 \mathrm{a}$ & $19.5 \mathrm{a}$ & $9.0 \mathrm{a}$ & $9.5 \mathrm{a}$ & $18.5 \mathrm{ab}$ & $10.0 \mathrm{a}$ & $11.0 \mathrm{a}$ & $19.0 \mathrm{a}$ \\
\hline Manfaloti & $0.0 \mathrm{~b}$ & $0.0 \mathrm{~d}$ & $19.0 \mathrm{a}$ & $0.5 \mathrm{~b}$ & $0.5 \mathrm{~b}$ & $19.0 \mathrm{a}$ & $0.5 \mathrm{~b}$ & $1.5 \mathrm{~cd}$ & $20.0 \mathrm{a}$ \\
\hline Banati & $0.0 \mathrm{~b}$ & $0.0 \mathrm{~d}$ & $18.0 \mathrm{a}$ & $1.0 \mathrm{~b}$ & $2.5 \mathrm{~b}$ & $11.0 \mathrm{c}$ & $1.0 \mathrm{~b}$ & $1.5 \mathrm{~cd}$ & $18.5 \mathrm{a}$ \\
\hline Succary & $0.5 \mathrm{ab}$ & $5.0 \mathrm{bc}$ & $20.0 \mathrm{a}$ & $2.0 \mathrm{~b}$ & $1.0 \mathrm{~b}$ & $11.0 \mathrm{c}$ & $3.5 \mathrm{~b}$ & $6.0 \mathrm{~b}$ & $17.0 \mathrm{a}$ \\
\hline
\end{tabular}

${ }^{2}$ Means (of three samples) separation in columns by Duncan's multiple range test, $\mathrm{P}=0.05$.

${ }^{y}$ Fresh pomegranates stored at 5C for 9 days.

Table 7. Chemical characteristics of pomegranate juice extracted from fresh fruits (F) or from fruits dried (D) at 30C and 33\% RH $(2.9$ $\mathrm{kpa}$ vapor pressure difference).

\begin{tabular}{|c|c|c|c|c|c|}
\hline Cultivar & $\mathrm{pH}$ & $\begin{array}{c}\mathrm{TSS}^{\mathrm{y}} \\
(\%)\end{array}$ & Acidity (\%) & $\begin{array}{c}\text { Vitamin C } \\
(\mathrm{mg} / 100 \mathrm{ml})\end{array}$ & $\begin{array}{c}\text { Total } \\
\text { sugar }(\%)\end{array}$ \\
\hline \multicolumn{6}{|l|}{ Taifi-A } \\
\hline $\mathbf{F}$ & $3.5 \mathrm{cde}$ & $16.3 \mathrm{a}$ & $0.5 \mathrm{cde}$ & $2.2 \mathrm{a}$ & $14.0 \mathrm{bcd}$ \\
\hline D & 3.7 . & 17.0 & 0.6 & 0.5 & 15.0 \\
\hline \multicolumn{6}{|l|}{ Taifi-R } \\
\hline F & $4.1 \mathrm{abc}$. & $15.4 \mathrm{abc}$ & $0.3 \mathrm{de}$ & $0.9 \mathrm{~d}$ & $14.2 \mathrm{a}-\mathrm{d}$ \\
\hline D & 4.2 & 16.8 & 0.4 & 0.2 & 16.0 \\
\hline \multicolumn{6}{|c|}{ El-Madina } \\
\hline $\mathrm{F}$ & $3.8 \mathrm{a}-\mathrm{e}$ & $16.0 \mathrm{ab}$ & $0.5 \mathrm{~cd}$ & $0.7 \mathrm{~d}$ & $14.6 \mathrm{abc}$ \\
\hline D & 3.8 & 16.8 & 0.6 & 0.2 & 12.5 \\
\hline \multicolumn{6}{|c|}{ Khad El-Jameel } \\
\hline $\mathbf{F}$ & $3.3 \mathrm{e}$ & $16.0 \mathrm{ab}$ & $1.4 \mathrm{a}$ & $1.6 \mathrm{~cd}$ & $14.4 \mathrm{abc}$ \\
\hline D & 3.8 & 17.0 & 1.6 & 0.5 & 13.1 \\
\hline \multicolumn{6}{|c|}{ Red Balady } \\
\hline F & $3.4 \mathrm{de}$ & $14.1 \mathrm{~d}$ & - $1.3 \mathrm{a}$ & $2.1 \mathrm{a}$ & $15.1 \mathrm{ab}$ \\
\hline D & 3.5 & 14.5 & 1.3 & 0.2 & 11.5 \\
\hline \multicolumn{6}{|c|}{ Mellasi } \\
\hline F & $3.6 \mathrm{~b}-\mathrm{e}$ & $15.3 \mathrm{bc}$ & $0.9 \mathrm{~b}$ & $2.2 \mathrm{a}$ & $15.2 \mathrm{a}$ \\
\hline D & 3.8 & 17.6 & 1.1 & 0.2 & 11.5 \\
\hline \multicolumn{6}{|c|}{ De-Jativa } \\
\hline $\mathbf{F}$ & $4.2 \mathrm{ab}$ & $14.2 \mathrm{~d}$ & $0.3 \mathrm{de}$ & $0.3 \mathrm{e}$ & $13.3 \mathrm{~d}$ \\
\hline $\mathrm{D}$ & 4.5 & 17.2 & 0.4 & 0.4 & 16.0 \\
\hline \multicolumn{6}{|l|}{ Molar } \\
\hline $\mathbf{F}$ & $4.0 \mathrm{a}-\mathrm{d}$ & $15.9 \mathrm{abc}$ & $0.5 \mathrm{c}$ & $0.3 \mathrm{e}$ & $13.8 \mathrm{~cd}$ \\
\hline D & 3.6 & 16.2 & 0.6 & 0.2 & 12.5 \\
\hline \multicolumn{6}{|c|}{ Manfaloti } \\
\hline $\mathbf{F}$ & $3.5 \mathrm{cde}$ & $15.8 \mathrm{abc}$ & $1.0 \mathrm{~b}$ & $1.4 \mathrm{c}$ & $13.8 \mathrm{~cd}$ \\
\hline D & 3.6 & 17.0 & 1.2 & 0.2 & 11.5 \\
\hline \multicolumn{6}{|l|}{ Banati } \\
\hline $\mathbf{F}$ & $3.6 \mathrm{~b}-\mathrm{e}$ & $15.2 \mathrm{bc}$ & $0.8 \mathrm{~b}$ & $1.7 \mathrm{~b}$ & $11.4 \mathrm{e}$ \\
\hline D & 3.6 & 15.6 & 1.3 & 0.6 & 13.9 \\
\hline \multicolumn{6}{|c|}{ Succary } \\
\hline $\mathrm{F}$ & $4.3 \mathrm{a}$ & $14.9 \mathrm{~cd}$ & $0.2 \mathrm{e}$ & $0.4 \mathrm{e}$ & $13.7 \mathrm{~cd}$ \\
\hline $\mathrm{D}$ & 3.8 & 16.8 & 0.5 & 0.4 & 16.0 \\
\hline$S E^{\mathbf{x}}$ & 0.26 & 0.39 & 0.12 & 0.11 & 0.45 \\
\hline
\end{tabular}

${ }^{z}$ Means separation in columns by Duncan's multiple range test, $P=$ 0.05 for fresh fruits only.

yTSS $=$ Total soluble solids concentration.

${ }^{\mathrm{x}} \mathrm{SE}=$ Standard error to compare interaction between cultivar and treatment.
Table 8. Shelf life ${ }^{z}$ and proportion of decayed fruity following drying for 9 days under the conditions indicated.

\begin{tabular}{|c|c|c|c|c|c|c|}
\hline \multirow[b]{3}{*}{ Cultivar } & \multicolumn{6}{|c|}{$\begin{array}{c}\text { Drying temp }\left({ }^{\circ} \mathrm{C}\right) / \mathrm{RH}(\%) \\
\text { [vapor pressure difference }(\mathrm{kPa})]\end{array}$} \\
\hline & \multicolumn{2}{|c|}{$20 / 47(1.2)$} & \multicolumn{2}{|c|}{$30 / 33(2.9)$} & \multicolumn{2}{|c|}{$40 / 25(5.5)$} \\
\hline & $\begin{array}{c}\text { Shelf } \\
\text { life } \\
\text { (days) }\end{array}$ & $\begin{array}{c}\text { Decayed } \\
\text { fruit } \\
(\%)\end{array}$ & $\begin{array}{c}\text { Shelf } \\
\text { life } \\
\text { (days) }\end{array}$ & $\begin{array}{c}\text { Decayed } \\
\text { fruit } \\
(\%) \\
\end{array}$ & $\begin{array}{c}\text { Shelf } \\
\text { life } \\
\text { (days) }\end{array}$ & $\begin{array}{c}\text { Decayed } \\
\text { fruit } \\
(\%) \\
\end{array}$ \\
\hline Taifi-A & 84 & 23.7 & 84 & 26.3 & 5 & 58.3 \\
\hline Taifi-R & 49 & 39.1 & 42 & 53.5 & 0 & 100 \\
\hline El-Madina & 91 & 22.8 & 119 & 23.5 & 5 & 36.6 \\
\hline Khab El-Jameel & 198 & 32.5 & 126 & 26.3 & 5 & 51.8 \\
\hline Red Balady & 91 & 21.9 & 119 & 20.1 & 5 & 41.1 \\
\hline Mellasi & 112 & 18.7 & 126 & 17.5 & 5 & 45.8 \\
\hline De-Jativa & 42 & 49.3 & 14 & 65.6 & 0 & 100 \\
\hline Molar & 49 & 43.8 & 14 & 58.7 & 0 & 100 \\
\hline Manfaloti & 84 & 22.8 & 126 & 16.2 & 5 & 35.6 \\
\hline Banati & 98 & 26.1 & 126 & 20.7 & 5 & 41.1 \\
\hline Succary & 35 & 39.4 & 14 & 49.6 & 0 & 100 \\
\hline
\end{tabular}

${ }^{2}$ Days until the dried pomegranates deteriorated to a general acceptability score of 3 or lower.

${ }^{y}$ Decay percentage at the end of indicated storage period.

transferred to room temperature where the first group had been kept. The following determinations were carried out during and/ or after drying: 1) Ten identified fruits from each group and for each cultivar were weighed daily during drying for 9 days. 2) External and internal symptoms on dried fruits were observed. Decayed fruits were discarded, and their cumulative percentages were calculated until the end of the drying period. 3) The compressive strength and compressive strain of dried fruits were measured as noted for the fresh fruits. 4) The same sensory evaluation panel used for evaluating the pomegranate cultivars was asked to detect attributable differences between fresh (coldstored) and dried pomegranates. 5) The extracted juice of dried fruits was analyzed for $\mathrm{pH}$, TSS, titratable acidity, vitamin $\mathrm{C}$, and reducing or nonreducing and total sugars (AOAC, 1980).

Storage of dried fruits. Dried fruits were stored at room temperature $(20 \pm 2 \mathrm{C})$, and the extent of shelf life of each cultivar was determined based on general acceptability on a 9-point heclonic scale, $(1-3=$ unacceptable; 4-6 = acceptable; 7-9 = excellent). Visual evaluation of quality was made every 5 days, 
while sensory evaluation was conducted every 2 weeks during storage. Chemical analyses were performed monthly (AOAC, 1980). The percentage of decayed fruits was calculated at the end of storage for each cultivar. Fungal mycelium was transferred to potato dextrose agar (Banwart, 1981), and, after incubation for 5 days at $25 \mathrm{C}$, molds were identified using the slide culture techniques of Dillello (1982).

\section{Results}

Evaluation of fresh fruits. The color of fresh fruits varied among cultivars from yellow ('Taifi-R', 'De-Jativa', 'Molar', 'Succary'), to greenish red ('Taifi-A'), to solid yellowish red ('El-Madina', 'Kab E1-Jameel', 'Manfaloti'), to bright red ('Mellasj', 'Banati'), to dark red ('Red Balady'). 'Manfaloti', 'Mellasi', and 'Kab E1-Jameel' weighed, on average, >300 g, while 'Taifi-A', 'Molar', and 'Succary' weighed < $200 \mathrm{~g}$ (Table 1). 'Kab El-Jameel', followed by 'Manfaloti', contained more edible portion and more juice than the other cultivars.

'Taifi-A' was given the highest sensory scores (Table 2). The other cultivars either lacked the attractive red pigmentation or the sweet taste, or they had a grainy mouth feel.

Weight loss and decay during drying. The pomegranate fruits gradually lost weight during drying under the various conditions (Table 3). Weight loss increased with increasing vapor pressure difference (higher temperatures, lower relative humidity). The drying pattern varied among cultivars, but most of the water was lost from the peel tissue rather than from the pulp (data not shown). Drying at 40C and 25\% RH severely damaged the fruits (Table 4), particularly those of 'Taifi-R', 'De-Jativa', 'Molar', and 'Succary', which softened on the 4th day of drying. The most prevalent types of damage other than softening were browning and superficial discoloration of the husk, mold-infected "scalded area, " and dripping leakage of juice. The fruits were much less affected by drying at $30 \mathrm{C}$ and $33 \% \mathrm{RH}$, and almost no fruits were discarded during drying at $20 \mathrm{C}$ and $47 \%$ $\mathrm{RH}$.

External and internal appearance of dried fruit. Upon drying, particularly at $40 \mathrm{C}$, the rind lost its natural color, shriveled, and became hard and dark. The dried fruits shrank toward the center and were irregular in shape. Internal symptoms of drying at $40 \mathrm{C}$ included splits, voids, and browning of the arils and white segments separating the arils. However, drying at 30 or 20C did not have any visually deleterious effect on the internal portion of the fruit.

Compressibilities of dried fruit. Two measures of compression were determined: 1) compressive strength $\left(\mathrm{kg} \cdot \mathrm{cm}^{-2}\right)$ $=$ force/contact area; 2 ) compressive strain $(\%)=$ total compressive distance/initial fruit height.

The compressive strength reflects the force required to crack the fruit, while compressive strain represents the deformation of the fruit until it cracks. The force necessary to cause cracking was generally higher for dried than for fresh fruit (Table 5), although the results were not always consistent. Drying resulted in harder rind that resisted cracking and in an irrigular shape that altered the size of the contact area between the compression plate and the equator of the fruit. This change may explain the difference in compressive strength between fresh and dried fruits. Drying caused a decrease in compressive strain of most fruits dried at $30 \mathrm{C}$ and $33 \% \mathrm{RH}$, or at $40 \mathrm{C}$ and $25 \% \mathrm{RH}$ (Table 5). Dried fruits had lost most of their natural elasticity during drying and were less tolerant to compression; consequently, shorter compressive distance was obtained. However, 'Taifi-R', 'DeJativa', and 'Succary' dried at 40C showed significantly (P <
0.05) longer total compressive distance (data not shown) and, consequently, higher compressive strain percentages, which is a symptom of softening of fruits during and after drying.

Sensory evaluation of dried fruit. Drying at 20C and $47 \%$ $\mathrm{RH}$ or at $30 \mathrm{C}$ and $33 \% \mathrm{RH}$ did not appear to have any deleterious effect on fruit quality (Table 6). However, a few panelists gave higher scores to refrigerated fruits than to fruits dried at $30 \mathrm{C}$ and $33 \% \mathrm{RH}$, while most gave the lowest scores to fruits dried at $40 \mathrm{C}$ and $25 \% \mathrm{RH}$ on the basis of their color, flavor, and overall acceptability. The effects of drying at 20C and $47 \%$ $\mathrm{RH}$ or at $30 \mathrm{C}$ and $33 \% \mathrm{RH}$ on sensory properties varied among cultivars; 'De-Jativa' and 'Molar' consistently were judged different from the refrigerated fruit (Table 6). Fruit dried at 40C and $25 \% \mathrm{RH}$ almost always was judged different from refrigerated fruit.

Chemical composition of fresh and dried fruits. 'Khab ElJameel' had the lowest $\mathrm{pH}$ and the highest-acidity, while 'Succary' had the highest pH (Table 7). Vitamin C content was highest and about equal in 'Taifi-A', 'Red Balady', and 'Mellasi'. TSS ranged from $14.1 \%$ to $16.3 \%$, while total sugar content ranged from $11.4 \%$ to $15.2 \%$. However, nonreducing sugars $(1.9 \%)$ were found only in 'Banati'.

After drying for 9 days at $30 \mathrm{C}$ and $33 \% \mathrm{RH}$, most fruits had generally higher $\mathrm{pH}$, TSS, and acidity but a lower vitamin $\mathrm{C}$ content than refrigerated fruits (Table 7). Drying had no consistent effect on total sugar content, and the reason for the inconsistency is unknown.

Drying at 20C and 47\% RH did not affect the chemical composition of the fruits (data not shown). The fruits dried at 40C and 25\% RH were damaged by drying and, therefore, their juice was not analyzed. The interaction for TSS, vitamin C, and total sugars between cultivar and treatment was significant $(\mathrm{P}<0.05)$ but not for $\mathrm{pH}$ and acidity.

Shelf life of dried fruits and their quality during storage. Fruits dried at $40 \mathrm{C}$ and then kept at $20 \pm 2 \mathrm{C}$ and $47 \%$ RH had a very short ( $\leq .5$ days) shelf life (Table 8 ). However, those dried at 20 or $30 \mathrm{C}$ remained acceptable for 14 to 126 days, depending on cultivar and drying conditions. Cultivars (De-Jativa, Molar, Succary, Taifi-R) that had been rapidly damaged by drying (Table 4$)$, had a shorter shelf life ( $<49$ days) than the others.

Fruits initially dried at $20 \mathrm{C}$ and $47 \% \mathrm{RH}$ showed some fungal decay during subsequent storage under the same conditions. The decay started as a soft area around the calyx and progressed as a mass of blackened arils inside the fruit. Aspergillus niger and some Penicillium spp., mainly Penicillium expansum Lk. ex Thorn. were identified (Raper and Fennel, 1965; Raper et al., 1968).

\section{Discussion}

The evaluation of fresh fruits showed the panelists' preference toward 'Taifi-A' over the other cultivars, including 'Taifi-R'. This result would be expected since 'Taifi-A' is the most popular cultivar in Saudi Arabia. 'Taifi-A' and 'Taifi-R' are grown in two completely different climatic regions, and the edible portion of 'Taifi-A' is a very attractive red, while that of 'Taifi-R' lacked this attribute. The mean fruit weight and edible portion of the fresh fruits are important from the economic standpoint whether the fruits are to be consumed fresh or used for juice extraction. Data presented here on 'Taifi' pomegranate are consistent with earlier studies (Ewaidah, 1987).

Weight loss during drying increased with temperature increase and relative humidity decrease. Elyatem and Kader (1984) 
found the weight loss of pomegranates during storage to be largely due to water loss; weight loss due to respiration represented only $\approx 9 \%$ to $26 \%$ of the total, depending on temperature. Weight loss of pomegranate fruits during storage at various temperatures was also reported by others (Al-Mughrabi and Bacha, 1986; Heikal et al., 1984). The change in color of the fruit caused by drying can reduce the marketability, while toughness of the rind can increase the durability during shipping and handling.

Drying at $30 \mathrm{C}$ and $33 \% \mathrm{RH}$ or at $20 \mathrm{C}$ and $47 \% \mathrm{RH}$ produced a brown, leathery, tough rind, but the edible portion remained acceptable and no internal changes were observed. However, the edible portion of fruits dried at 40C browned and had a poor flavor. The browning of plant tissues may be due to either enzymatic or nonenzymatic and both were investigated by Ben Arie and Or (1986) in 'Wonderful' pomegranate. They suggested that discoloration was a result of enzymatic oxidation.

The increase in pH, TSS, and acidity and the decrease in vitamin $\mathrm{C}$ upon drying of pomegranate cultivars are in agreement with the findings of other workers (Al-Mughrabi and Bucha, 1986; Elyatem and Kader, 1984; Heikal et al., 1984).

The shelf life of dried pomegranates varied among cultivars, depending on drying conditions. Fruits dried at 30C and $33 \%$ $\mathrm{RH}$ or at $20 \mathrm{C}$ and $47 \% \mathrm{RH}$ had better keeping quality ( $>3$ months) than those dried at 40C and $25 \%$ RH, which deteriorated rapidly. Al-Mughrabi and Bucha (1986) reported that $21.46 \%, 13.72 \%$, and $24.82 \%$ of fruit were decayed for 'Manfaloti', 'Mellasi', and 'Khab El-Jameel', respectively, after storage for 10 weeks at room temperature (20 to 30C). Elyatem and Kader (1984) discarded 'Wonderful' pomegranate fruits after 6, 4 , and 1 weeks of storage at 10,20 , or $30 \mathrm{C}$, respectively, because of decay. However, unlike in this study they had not treated the fruits with postharvest fungicides.

Partial drying of pomegranates can be a successful method of preservation if drying conditions are properly chosen and carefully controlled. The results of this study suggest that drying temperature should not exceed 30C to minimize the deleterious effect on quality. Partially dried pomegranates can have slightly inferior eating quality than those refrigerated, but they might find some applications in the manufacture of jams, jellies, and other products.

\section{Literature Cited}

Al-Mughrabi, M.A. and M.A. Bacha. 1986. Effect of postharvest application of GA, 2, 4-D and cold storage on keeping quality of pomegranate fruits. J. College Agr., King Saud Univ. 8:143-154.

AOAC. 1980. Official methods of analysis. 15th ed. Assn. Offic. Anal. Chemists, Washington, D.C.

Banwart, G.J. 1981. Basic food microbiology. AVI, Westport, Corm.

Ben-Arie, R. and E. Or. 1986. The development and control of husk scald on 'Wonderful' pomegranate fruit during storage. J. Amer. Soc. Hort. Sci. 111:395-399.

Chase, E. M., C.G. Church, and H.D. Poore. 1930. The 'Wonderful' variety of pomegranate. U.S. Dept. Agr. Circ. 98.

Diliello. L.R. 1982. Methods in food and dairy microbiology. AVI, Westport, Conn.

Elyatem, M.S. and A.A. Kader. 1984. Post-harvest physiology and storage behavior of pomegranate fruits. Scientia Hort. 24:287-298.

Ewaidah, E.H. 1987. Nutrient composition of "Taifi" pomegranate (Punica granatum L.) fragments and their suitability for the production of jam. Arab Gulf J. Sci. Res. Agr. Biol. Sci. B5(3):367-378.

Heikal, H.A., M.M. Ismail, and S. Elyatem. 1984. Studies on storage of pomegranate. Agr. Res. Rev. (Cairo) 44(3):86-96.

Hodgson, R.W. 1917. The pomegranate. Calif. Agr. Expt. Sta. Bul. 276. p. 163-192.

Kader, A.A., A. Chordas, and S. Elyatem. 1984. Responses of pomegranate to ethylene treatment and storage temperature. Calif. Agr. 38(7-8):14-15.

LaRue, J.H. 1969. Growing pomegranate in California Univ. of Calif. Agr. Expt. Sta. Lflt 305.

Lutz, J.M. and R.E. Hardenburg. 1977. The commercial storage of fruits, vegetables, and florist and nursery stocks. U.S. Dept. Agr., Agr. Hdbk. 66.

Mukerjee, P.K. 1958. Storage of pomegranates (Punica granatum L.) Sci. Cult. 24:94.

Naser, T.A. 1983. Evergreen and deciduous fruits production and important cultivars in the Arab world. 1st ed. Dar Alma'arif, Cairo.

Pantastico, E.B., T.K. Chattopadhyay, and H. Subramanyam. 1975. Storage and commercial storage operations, p. 314-338. In: E.B. Pantastico (cd.). Postharvest physiology, handling and utilization of tropical and subtropical fruits and vegetables. AVI, Westport, Corm.

Raper, K.B. and 0.I. Fennell. 1965. The genus Aspergillus. Wavely Press, Baltimore.

Raper, K. B., C. Thorn, and D.I. Fennell. 1968. A manual of the Penicillin. Hafner, New York.

Shaheen, M.A. 1985. Suitability of some introduced pomegranate cultivars for Riyadh Region. J. College Agr., King Saud Univ. 7:147-152. 